Award Reference Number DE-FG03-00SF22021

Final Report

\title{
Supernova Hydrodynamics on the Omega Laser
}

\author{
National Laser Users' Facility \\ Department of Energy \\ Oakland Operations Office \\ 1301 Clay Street
}

Oakland, California 94612-5208

\section{Principal Investigators:}

\author{
R. Paul Drake \\ University of Michigan \\ Bruce Remington \\ Center for Laser Astrophysics-ILSA, LLNL
}

\section{Co-Investigators:}

Gail Glendinning

Center for Laser Astrophysics-ILSA; LLNL

Kim Budil

LLNL

Harry Robey

$L L N L$

Romain Teyssier

CEA Saclay, France

Omar Hurricane

LLNL

Ted Perry

LLNL

Dimitri Ryutov

LLNL

Jim Knauer

LLE; University of Rochester

Keisuke Shigemori Osaka University; LLNL

John Grove LANL

Grant Bazan

LLNL
Dave Arnett

University of Arizona

Jave Kane

University of Arizona; LLNL

Dick McCray

University of Colorado

Robert Rosner

University of Chicago

Bruce Fryxell

University of Chicago

Christof Litwin

University of Chicago

Adam Frank

University of Rochester

James Carroll

Eastern Michigan University

James Glimm

State University of New York-Stony Brook

Mary Jane Graham

West Point

Riccardo Betti

University of Rochester, Massachusetts Institute of Technology

November 15, 2001 


\section{Technical Report}

We have provided the following brief summary of our activities. We will be pleased to provide further information to anyone who requests it.

Supernovae are not well understood. Recent observations have clarified the depth of our ignorance, by producing observed phenomena that current theory and computer simulations cannot reproduce. Such theories and simulations involve, however, a number of physical mechanisms that have never been studied in isolation. In this project, which involved 22 Co-Investigators from 11 institutions, the Omega laser was used to conduct well-scaled experiments that investigate such mechanisms. Such experiments also provide clear tests of the codes used to simulate astrophysical phenomena. We are working to develop an experimental astrophysics testbed for compressible

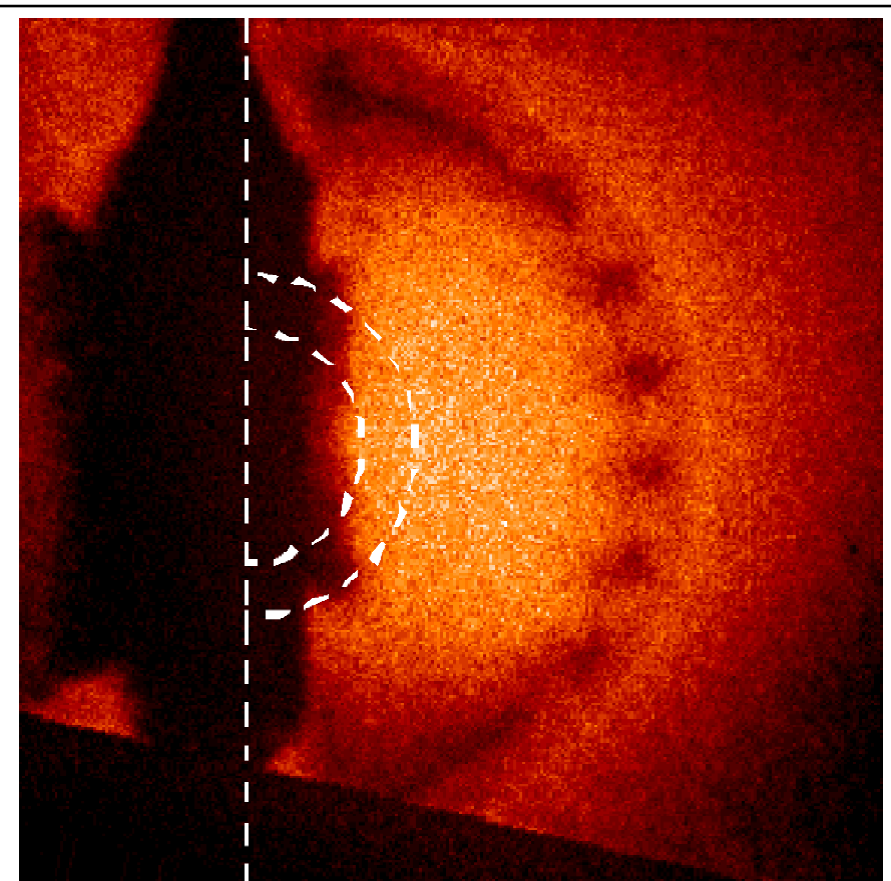

Figure 1. X-ray radiograph, showing modulations on a spherical capsule that has expanded into resorcinol foam of density $100 \mathrm{mg} / \mathrm{cm}^{3}$. The initial outside diameter of the Br-doped $\mathrm{CH}$ capsule was $532 \mu \mathrm{m}$ and the wall thickness was $97 \mu \mathrm{m}$. The initial capsule location is shown by white dashed lines. Initial perturbation wavelength and amplitude were $70 \mu \mathrm{m}$ and $10 \mu \mathrm{m}$, respectively. Hydrodynamic instabilities, like those present in supernovae, caused the observed modulations to develop from small initial perturbations hydrodynamics relevant to supernovae. The necessity for strong compression/decompression, strong shocks (Mach $\sim 10$ or greater), flexible geometries, and very smooth laser beams means that the 60-beam Omega laser is the only facility capable of carrying out this research program. In experiments at the Omega laser, and in related simulations, we have focused during this project on several interrelated aspects of this problem. We have now completed our studies of two aspects: 1) the evolution of the nonlinear RayleighTaylor (RT) and Richtmyer-Meshkov (RM) instabilities in divergent geometry, and 2) the effect structure at one interface can have on a second interface. Figures 1 and 2 show images from these experiments. We have begun, and are continuing, studies of two other aspects: 3 ) the production of a radiative precursor shock, and 4) the 3D vs. 2D evolution of 
the RM and RT instabilities in planar geometry at a decelerating interface.

These experiments have been quite successful. At Division of Plasma Physics meetings of the American Physical Society in 2000 and 2001, we presented 1 review paper, 1 invited talk, 1 tutorial, and 5 contributed papers. We also presented a number of papers at the conference on Laboratory Astrophysics with Large Lasers, at the American Astronomical Society meeting, and at the Anomalous Absorption Conference. The invited presentations at the APS meeting summarized our progress in creating an astrophysical testbed and in developing general relations for scaling from the laboratory to astrophysics. Drake and Remington were both asked to speak at the meeting of the NRC "Committee on the Physics of the Universe", and both presented invited talks at the IFSA meeting in Japan in September 2001. In addition, Discover magazine featured our work at Omega in its June, 2001 cover story "Star in a Jar", and last December, during our experiments,
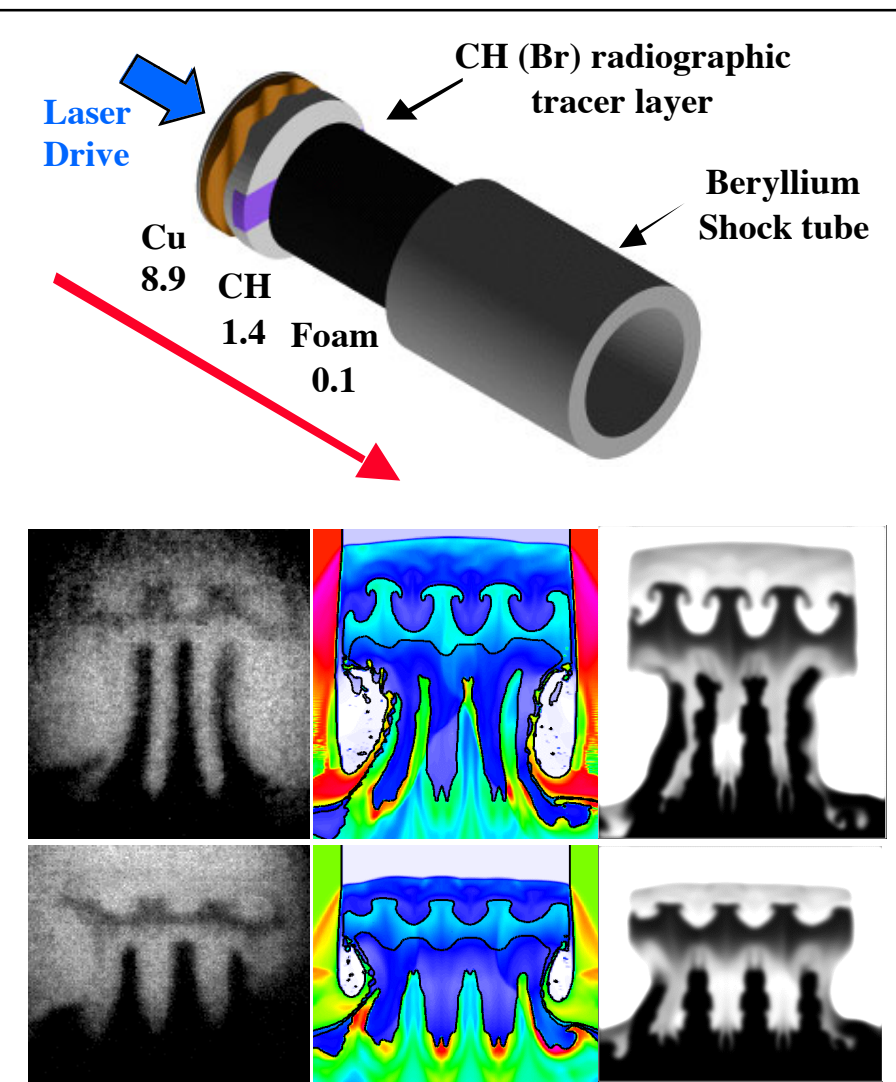

Figure 2. The interface coupling experiment. The target structure is shown at the top. The lower and upper images are at $39 \mathrm{~ns}$ and $65 \mathrm{~ns}$, respectively. The left images are data; the others are simulated radiographs from CALE (middle) and PROMETHEUS (right). the $\mathrm{BBC}$ came to Omega to film footage for a documentary on the origin of the elements. We have eight papers for publication that are in print, in press, or submitted, as listed below. The analysis and writing of these publications occupied most of our effort under this grant during its extension. We also used the experiments and the analysis as an opportunity to give four undergraduate students the experience of attending and participating in a large-scale science experiment.

In addition, our collaborators have produced simulations of these experiments. The group in Chicago has simulated the coupled interface experiments, and will simulate the $2 \mathrm{D}$ vs 3D experiments. The group at Stony Brook, with collaboration at Los Alamos, has simulated the diverging 
experiments and will simulate the multimode experiments we are planning for the future. The group at Maryland has simulated the radiative precursor experiments and will be involved in their interpretation.

\section{Publications based on this work.}

\section{In print:}

Kane, J.O., H.F. Robey, B.A. Remington, R.P. Drake, J. Knauer, D.D. Ryutov, H. Louis, R. Teyssier, O. Hurricane, D. Arnett, R. Rosner, and A. Calder, Interface imprinting by a rippled shock using an intense laser, Phys. Rev. E, 63 (May), 055401R, 2001.

Remington, B.A., R.P. Drake, D. Arnett, and H. Takabe, A Review of Astrophysics Experiments on Intense Lasers, Physics of Plasmas, 7 (May), 1641, 2000.

Robey, H.F., J.O. Kane, B.A. Remington, R.P. Drake, O.A. Hurricane, H. Louis, R.J. Wallace, J. Knauer, P. Keiter, D. Arnett, and D.D. Ryutov, An experimental testbed for the study of hydrodynamic issues in supernovae, Phys. Plasmas, 8 (May), 2446-2453, 2001.

Ryutov, D.D., B.A. Remington, H.F. Robey, and R.P. Drake, Magnetohydrodynamic scaling: from astrophysics to the laboratory, Phys. Plasmas, 8, 1804-1816, 2001.

\section{In press:}

Drake, R.P., and P.A. Keiter, Rayleigh Taylor growth at decelerating interfaces, Phys. Plasmas, in press.

Drake, R.P., H.F. Robey, O.A. Hurricane, Y. Zhang, B.A. Remington, J. Knauer, J. Glimm, D. Arnett, J.O. Kane, K.S. Budil, and J. Grove, Experiments to produce a hydrodynamically unstable, spherically diverging system of relevance to instabilities in supernovae, Astrophys. J., in press.

\section{Submitted:}

Keiter, P.A., R.P. Drake, T.S. Perry, H.F. Robey, B.A. Remington, C.A. Iglesias, R.J. Wallace, and J. Knauer, Observation of a hydrodynamically-driven, radiative-precursor shock, Phys. Rev. Lett., submitted, 2001.

Calder, A., B. Fryxell, T. Plewa, R. Rosner, T. Dupont, H.F. Robey, J.O. Kane, B.A. Remington, R.P. Drake, G. Dimonte, M. Zingale, L.J. Dursi, F.X. Timmes, K. Olson, P. Ricker, P. MacNeice, and H.M. Tufo, On validating an astrophysical simulation code, Astrophys. J., submitted, 2001. 